\title{
Stance and weight distribution after tibial plateau leveling osteotomy in forelimb and hind limb amputee dogs
}

Ron Ben-Amotz ${ }^{1,2^{*}}$ D, David Dycus ${ }^{3}$, David Levine ${ }^{4}$, Andréia Gonçalves Arruda ${ }^{5}$, Nicholas Fagan ${ }^{6}$ and Denis Marcellin-Little

\begin{abstract}
Background: Little is known about the weight distribution to the remaining limbs for amputee dogs that undergo orthopedic surgery.

The objective of the paper was to describe stance and weight distribution after tibial plateau leveling osteotomy (TPLO) in forelimb and in hind limb amputees (AmpTPLO) and to compare them to four-legged TPLO patients (4LTPLO) and amputees without TPLO (Amp). Weight bearing distribution at a stance was compared between groups. Joint angles of forelimb and hind limb joints in a sagittal plane, hind limb orientation in a frontal plane, and pelvic orientation in a transverse plane (pelvic tilt) were measured and compared between groups.

Results: Joint angles, hind limb abduction, and pelvic tilt of AmpTPLO and Amp did not differ statistically. Mean weight bearing in the operated hind limb was higher for AmPTPLO than $4 \mathrm{~L}_{\text {TPLO }}$. Mean weight bearing for thoracic limbs of AmpTPLO and $4 \mathrm{~L}_{\text {TPLO }}$ did not differ statistically. Weight bearing of the hind limb of AmpTPLO and Amp did not differ statistically.
\end{abstract}

Conclusions: The position of the center of mass and posture of Amp TPLO and Amp does not differ. The weight distribution and posture of Amp is not impacted negatively by TPLO.

Keywords: Dog, Amputation, Tibial plateau leveling, TPLO, Weight distribution

\section{Background}

Amputation of a limb is performed to manage complex fractures, neoplasia, osteomyelitis, soft tissue infection, and limb deformities that cause severe disability in dogs [1]. While recovery after thoracic limb or hind limb amputation is satisfactory in most instances the posture and mobility of amputee dogs vary widely [1-3]. The loss of a limb results in an increase in the ground reaction forces and contact times of the remaining limbs. These increases in ground reaction forces and contact

\footnotetext{
* Correspondence: ronba1@gmail.com

${ }^{1}$ Small Animal Orthopedics, Koret School of Veterinary Medicine, Rehovot, Israel

${ }^{2}$ The Hebrew University of Jerusalem, P.O Box 12, 76100 Rehovot, Israel Full list of author information is available at the end of the article
}

times are greater after the loss of a forelimb than a hind limb $[4,5]$. Hind limb amputee dogs also adjust to the loss of a hind limb by increasing tarsal joint range of motion as well as increase range of the cervicothoracic and thoracolumbar vertebral regions [6]. A significant dilemma whether an amputee dog that develops a major orthopedic disease such as CCL rupture, can have an acceptable limb function following an orthopedic repair. Rupture of the cranial cruciate ligament (CCL) and the subsequent development of progressive osteoarthritis is the most common cause of hind limb lameness in dogs. Osteotomy procedures such as the tibial plateau leveling osteotomy (TPLO), are commonly recommended [7]. 
Objective clinical information related to recovery after TPLO is lacking. To the authors' knowledge, only one scientific article describes patient outcomes and owner satisfaction for dogs undergoing orthopedic surgery after an amputation [8]. In that article, 11 amputees had a CCL rupture and nine of those underwent TPLO. The survey respondents were satisfied with outcome of amputee dogs that had a TPLO surgery. However, information describing stance and the position of the center of mass in amputees after surgical stabilization following CCL rupture has not been reported in the literature.

The objectives of the current study were to measure the weight distribution and posture of forelimb and hind

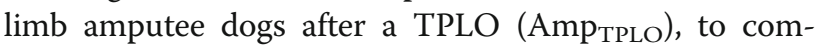
pare those to weight distribution and posture in amputee dogs not undergoing a TPLO (Amp) and to fourlegged dogs after a TPLO (4L $\left.\mathrm{L}_{\mathrm{TPLO}}\right)$. We hypothesized

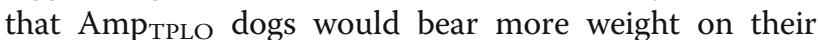
remaining hind limb than $4 \mathrm{~L}_{\text {TPLO }}$ and would bear less weight on their operated leg than Amp. We also hypoth-

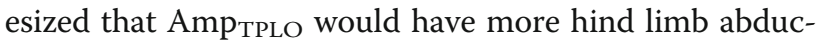
tion and pelvic tilt than $4 \mathrm{~L}_{\mathrm{TPLO}}$.

\section{Results}

Twenty-eight dogs were enrolled in the study: seven Amp Tplo, $10 \mathrm{Amp}$, and $114 \mathrm{~L}_{\mathrm{TPlO}}$. Among Amp, eight dogs had a hind limb amputation and two dogs had a thoracic limb amputation. For Amp ${ }_{\text {TPLO }}$, four dogs had a thoracic limb amputation, and three dogs had a hind limb amputation. For $4 \mathrm{~L}_{\text {TPLO }}$ three TPLOs were performed on the left limb and eight in the right limb. Detailed information for dogs included in this case series is presented on Table 1. The mean age for Amp TPLO $_{\text {TP }}$ at the time of surgery was 7.0 years (range, 1.5 to 12.6 years) and mean weight was $19.1 \pm 12.9 \mathrm{~kg}$. Four males (57\%) and three females (43\%) were included. The mean age for Amp was 9.8 years (range, 2.0 to 13.1 years) and mean weight was $21.8 \pm 6.7 \mathrm{~kg}$. Seven females $(70 \%)$ and three males $(30 \%)$ were included. The mean age for the $4 \mathrm{~L}_{\text {TPLO }}$ was 5.6 years (range, 3.3 to 8.9 years) and mean weight was $22.9 \pm 10.8 \mathrm{~kg}$. Six females (54\%) and five males (45\%) were included. Dog breeds are reported in Table 1.

The weight distribution among Amp ${ }_{\text {TPLO }}$, Amp, and $4 \mathrm{~L}_{\text {TPLO }}$ dogs did not differ statistically $(P=0.56,0.50$, and 0.94 , respectively for comparison among groups).

Joint angles of the thoracic limbs including shoulder, elbow, and carpus in Amp тPLO and Amp did not differ statistically $\left(107.6^{\circ} \pm 10.2^{\circ}\right.$ and $99.9^{\circ} \pm 13.3^{\circ}, P=0.60$; $123.3^{\circ} \pm 13.9^{\circ}$ and $125.0^{\circ} \pm 13.4^{\circ}, P=0.91$; and $130.55^{\circ} \pm$ $13.83^{\circ}, 135.30^{\circ} \pm 16.29^{\circ}, P=0.73$, respectively). Similarly, hind limb joint angle measurements including hip, stifle and tarsal angles in Amp TPLO and Amp did not differ statistically $\left(114.8^{\circ} \pm 13.5^{\circ}\right.$ and $110.4^{\circ} \pm 13.1^{\circ}, P=0.49$; $154.7^{\circ} \pm 8.7^{\circ}$ and $145.7^{\circ} \pm 10.9^{\circ}, P=0.42$; and $210.5^{\circ} \pm 6.7^{\circ}$ and $217.9^{\circ} \pm 11.6^{\circ}, P=0.82$, respectively). There was no statistical difference between pelvic tilt and limb adduction among $\mathrm{Amp}_{\text {TPLO }}$ and Amp $(P=0.56$ and 0.45 , respectively). The mean \pm SD percentage weight bearing in the operated limb was higher in Amp TPLO $(27.1 \pm 3.3 \%$, range, 23.0 to $32.1 \%)$ than $4 \mathrm{~L}_{\mathrm{TPLO}}(15.5 \pm 2.1 \%$, range, 11.9 to $18.8 \%, P<0.001)$. The percentage weight distribution in the hind limb for Amp TPLO $(29.1 \pm 2.8 \%$, range 26.5 to $32.1 \%)$ and Amp $(26.4 \pm 3.4 \%$, range 21.3 to $32.0 \%)$ did not differ $(P=0.22)$; (Fig. 1$)$. The mean percentage weight bearing in thoracic limbs did not differ between Amp тPLO $(61.4 \pm 4.1 \%)$ and $4 \mathrm{~L}_{\mathrm{TPLO}}(57.1 \pm$ $13.1 \%, P=0.62$ ).

\section{Discussion}

The purpose of this study was to identify and report objective data pertaining to amputee dogs with CCL injury that have undergone a TPLO procedure. The decision to proceed with surgical stabilization of the affected stifle in these animals may be challenging for both clinicians and owners, particularly when an osteotomy procedure is recommended. The authors believe that the perception of a challenging recovery and undesirable outcome may result in many of these cases remaining untreated. Therefore, the data presented in this paper has the potential to assist both clinicians and owners in the decision-making process. The objectives of the current study were to describe stance and weight distribution in Amp TPLO $_{\text {and }}$ an compare posture (joint angle, pelvic tilt, limb varus), weight distribution, and weight shift, between $\mathrm{Amp}_{\mathrm{T}}$ PLO, and two other groups of dogs: Amp and $4 \mathrm{~L}_{\text {TPLO }}$. We rejected the hypothesis that the posture of Amp PLO differed from Amp. The lack of postural differences between Amp ${ }_{\text {TPLO }}$ and Amp may inform the decisions of owners and clinicians considering TPLO to manage CCL injury in an amputee. We rejected the hypothesis that Amp TPLO $_{\text {would shift more weight }}$ to their thoracic limbs compared to Amp as there was no difference in weight distribution to the thoracic limbs between the two groups. Amp TPLO $_{\text {appear }}$ to re-establish appropriate thoracic limb weight bearing over a relatively short period of time. When com-

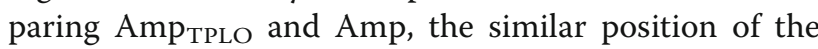
center of mass position, confirms the fact that posture is not impacted by TPLO in amputees. We rejected the hypothesis that Amp less weight on their operated limbs compared to Amp. These results suggest a positive response to TPLO on the remaining limb.

Kinetic and kinematic gait analysis has been shown to be more objective than visual examination in the detection of lameness and in evaluating limb function $[9,10]$. 
Table 1 Demographic data for 7 amputees who underwent a TPLO (AmptPLo), 10 control amputees (Amp), and 11 control fourlegged dogs (4LTPLO) who underwent a TPLO

\begin{tabular}{|c|c|c|c|c|c|c|}
\hline Group & Age (years) & Weight (kg) & Sex & Breed & Amputated limb & Side of TPLO \\
\hline Amp TPLO & 12.6 & 6.5 & $\mathrm{~F}$ & Chihuahua mix & $\mathrm{RF}$ & $R$ \\
\hline AmptPLO & 8.0 & 8.6 & M & Pomeranian mix & $\mathrm{RR}$ & L \\
\hline Amp TPLO & 1.3 & 5.0 & $\mathrm{~F}$ & Miniature poodle & LF & L \\
\hline Amp TPLO & 6.0 & 30.1 & M & Pitbull & RR & R \\
\hline AmptPLO & 7.0 & 40.0 & M & German SD & LF & $\mathrm{R}$ \\
\hline AmptPLO & 9.9 & 21.4 & M & Mix & $L R$ & R \\
\hline AmptPLO & 4.0 & 22.7 & $\mathrm{~F}$ & German SD & RF & $\mathrm{R}$ \\
\hline Amp & 10.1 & 10.3 & $\mathrm{~F}$ & American Eskimo & RR & - \\
\hline Amp & 13.1 & 22.1 & $\mathrm{~F}$ & Pointer & RR & - \\
\hline Amp & 10.8 & 21.5 & $\mathrm{~F}$ & Pitbull & $\mathrm{RR}$ & - \\
\hline Amp & 10.3 & 17.1 & $\mathrm{~F}$ & Mix & LR & - \\
\hline Amp & 11.4 & 30.4 & M & Golden retriever & RR & - \\
\hline Amp & 9.0 & 22.3 & $\mathrm{~F}$ & Mix & RR & - \\
\hline Amp & 18.0 & 20.0 & $\mathrm{~F}$ & German SP & $\mathrm{RF}$ & - \\
\hline Amp & 2.0 & 34.0 & $\mathrm{~F}$ & Husky mix & LR & - \\
\hline Amp & 9.0 & 24.0 & M & Bull terrier & RR & - \\
\hline Amp & 4.0 & 17.0 & M & Beagle & LF & - \\
\hline $4 \mathrm{~L}_{\mathrm{TPLO}}$ & 4.8 & 22.7 & $\mathrm{~F}$ & Am Staffordshire & - & $\mathrm{R}$ \\
\hline $4 \mathrm{~L}_{\mathrm{TPLO}}$ & 3.3 & 28.1 & M & Labrador retriever & - & $\mathrm{R}$ \\
\hline $4 \mathrm{~L}_{\mathrm{TPLO}}$ & 7.0 & 13.1 & M & Mix & - & $\mathrm{R}$ \\
\hline $4 \mathrm{~L}_{\mathrm{TPLO}}$ & 3.4 & 32.3 & M & Labrador retriever & - & L \\
\hline $4 \mathrm{~L}_{\mathrm{TPLO}}$ & 4.4 & 41.9 & $\mathrm{~F}$ & Great Pyrenees & - & L \\
\hline $4 \mathrm{~L}_{\mathrm{TPLO}}$ & 5.5 & 7.7 & $\mathrm{~F}$ & Poodle $x$ pug mix & - & R \\
\hline $4 \mathrm{~L}_{\mathrm{TPLO}}$ & 6.0 & 36.4 & $\mathrm{~F}$ & Bouvier des Flandres & - & $R$ \\
\hline $4 \mathrm{~L}_{\mathrm{TPLO}}$ & 7.9 & 14.0 & M & Cocker spaniel & - & R \\
\hline $4 \mathrm{~L}_{\mathrm{TPLO}}$ & 8.0 & 21.3 & M & Pitbull & - & L \\
\hline $4 \mathrm{~L}_{\mathrm{TPLO}}$ & 2.6 & 19.2 & $\mathrm{~F}$ & Shepherd mix & - & R \\
\hline $4 \mathrm{~L}_{\mathrm{TPLO}}$ & 8.9 & 17.3 & $\mathrm{~F}$ & Mix & - & $\mathrm{R}$ \\
\hline
\end{tabular}

Abbreviations: SD Shepherd dog; SP Shorthaired pointer; Am Amercian; $R F$ Right forelimb; $L F$ Left forelimb; $R R$ Right hind limb; $L R$ Left hind limb; $R$ Right; $L$ Left

Kinetic and kinematic studies have been completed to give objective data to pet owners as to how dogs will adapt following an amputation [6, 11, 12]. Kinetic changes after amputation indicate that amputee dogs use a compensatory mechanism that involves the unaffected diagonal limb pair $[6,12]$.

We selected a weight distribution platform in the current study since amputee patients may not be able to reliably ambulate at the required velocity to obtain valid trials on force plate or pressure sensitive walkways. In addition, weight distribution platforms are less costly than force plate and pressures sensitive walkways, require less space, and require fewer skills during data acquisition [13-15]. We accepted the hy-

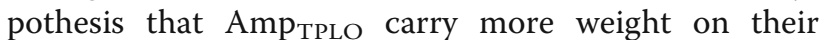
operated limb compared to $4 \mathrm{~L}_{\mathrm{TPLO}}$. In a recent article describing the long-term outcome of $4 \mathrm{~L}_{\mathrm{TPLO}}$ using force plate analysis, symmetry indices of the TPLO group did not differ from a normal control group 6 to 12 months after surgery [16].

When evaluating the effects of limb amputation on standing weight distribution in the remaining three limbs, dogs with a previous hind limb amputation had the largest mean increase in weight bearing in the contralateral thoracic limb [14]. Interestingly, our results suggest that Amp TPLO increase the weight placed on their ipsilateral thoracic limb. In contrast to four-legged control dogs which bear $60 \%$ of their weight in the forelimbs and $40 \%$ in the hind limbs [11]. After a hind limb amputation, dogs bear $74 \%$ of their weight on their thoracic limbs and $26 \%$ in the remaining hind limb [5]. In the current study, 


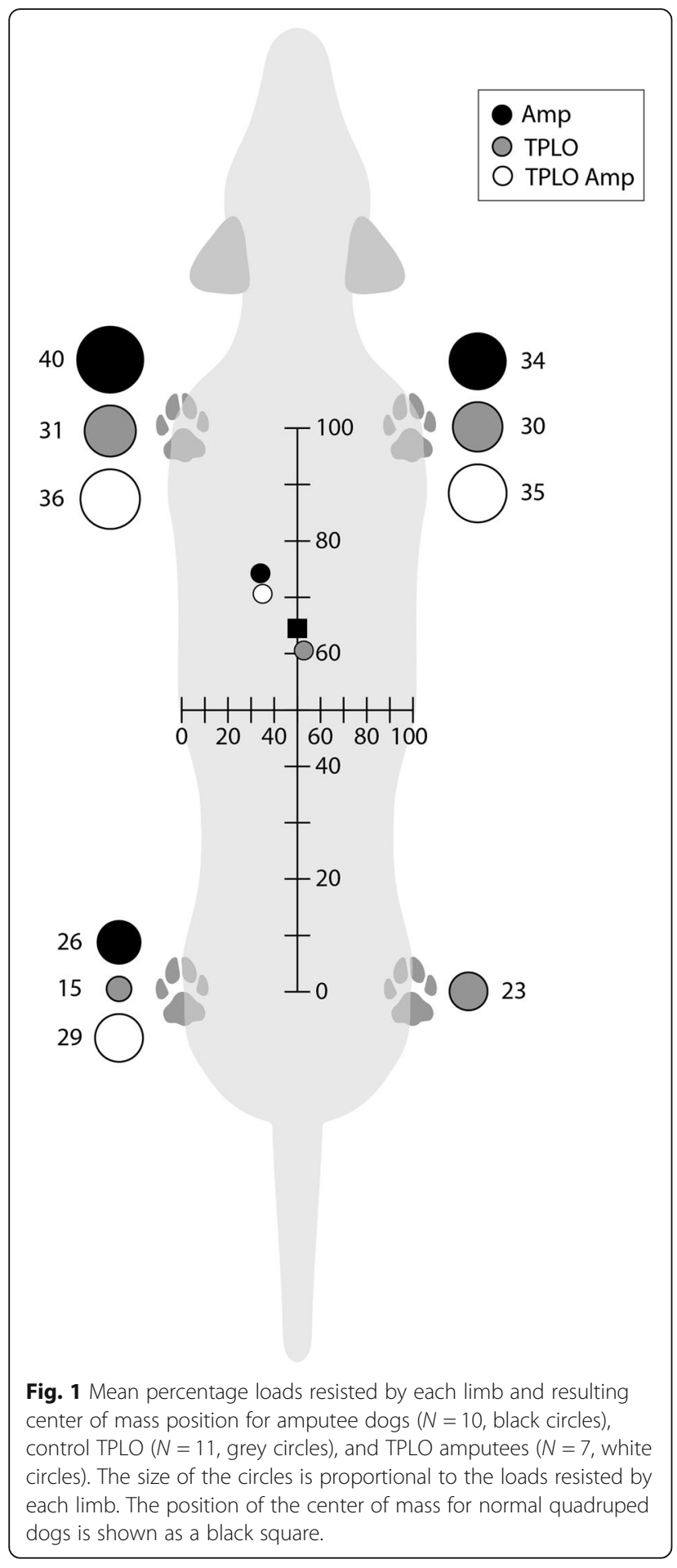

Amp TPLO appear to behave similarly to those Amp with regards to thoracic limb weight distribution.

When facing a cranial cruciate CCL ligament injury in Amp, a TPLO appears to be an appropriate treatment option because changes in posture or weight distribution after surgery are not expected. Proactive monitoring of orthopedic disease in the front limbs may be advisable in dogs with a previous limb amputation that undergo a surgical repair such as a TPLO. In addition, when determining candidacy for repair of the remaining hind limb, disease of the contralateral forelimb should be thoroughly evaluated.

This study had limitations: The procedure was performed by several surgeons. Data before TPLO were not available, the small sample size could have prevented enough power to detect potential statistical associations, the variability among forelimb and hind limb amputee patients within the Amp TPLO $_{\text {and Amp. }}$ and Both forelimb and hind limb amputees that had undergone a TPLO had acceptable weight bearing on their operated limbs with minimal postural difference compared to Amp. Since the current study was limited to an evaluation of stance, it is unclear how the gait at a walk and trot of forelimb and hind limb amputees that underwent a TPLO were impacted by the combination of an amputation and a TPLO. The gait adaptations of forelimb and hind limb amputees at trot have been described $[6,12]$ and are complex. Briefly, in forelimb amputees, the ipsilateral hind limb assume the role of a forelimb and a hind limb [12]. In pelvic limb amputees, changes affect mainly the contralateral tarsus and the cervico-thoracic and lumbosacral vertebral regions [6]. Further work should be performed to determine what long-term changes occur with weight bearing in amputee dogs undergoing a TPLO. Joint angles were measured by placing dots on anatomic landmarks and measuring angles between lines formed by connecting these dots. The method used had good repeatability in a previous study [17]. However, its accuracy is not known. The relatively small differences in joint angles among groups appeared to be proportional to the small difference in position of the center of mass, indirectly suggesting that the measurements were accurate. Although kinetic and kinematic analysis provide more objective data regarding weight bearing in dogs compared to visual observation, stance analysis may be a more readily available modality and may be easier to use.

\section{Conclusion}

In conclusion, a TPLO procedure in amputees results in an acceptable weight bearing with minimal postural difference compared to Amp.

\section{Methods}

Dog sample

All dogs undergo either a forequarter amputation or a coxofemoral disarticulation at least 8 weeks or more prior to being enrolled in the study. Patients treated at three veterinary specialty practices ${ }^{1,2,3}$ for complete CCL 
tear during the study period were eligible for inclusion if they were Amp ${ }_{\mathrm{TPLO}}$, Amp, or $4 \mathrm{~L}_{\mathrm{TPLO}}$, if owners signed an informed consent, and if a follow-up 8 weeks or more after TPLO was performed. No meniscal injuries were identified during surgery. TPLO were stabilized with a bone plate and a combination of locking and nonlocking screws. ${ }^{4}$ Craniocaudal and mediolateral stifle radiographs were acquired before surgery, immediately after surgery, and 8 weeks or more after surgery. Dogs were excluded from the study if an orthopedic disease problem other than CCL injury was detected during the orthopedic evaluation.

\section{Data collected}

Data collected included breed, sex, age at the time of surgery, limb amputated, presenting complaint, physical examination and orthopedic findings, the presence or absence of pre-existing joint disease, complications, and outcome.

Standing weight distribution was measured using a weight distribution platform. ${ }^{5}$ Dogs stood on the platform naturally, with their head and neck facing forward. For each patient, fifteen (on and off the platform) measurements of weight distribution were recorded and means were calculated. Photographs of the left and right sides, back and front of each dog were acquired using a camera placed at the level of stifle or elbow joint (Fig. 2). The camera ${ }^{6}$ was placed $2 \mathrm{~m}$ from the dog and was perpendicular to the long axis of the dog. For dogs with long hair, the hair was clipped to aid in identification of anatomic landmarks.

Shoulder, elbow, carpus, hip, stifle, and tarsus angles were measured in a sagittal plane, hind limb alignment in a frontal plane (abduction or adduction), and pelvic orientation in a transverse plane (pelvic tilt) were measured using a previously established protocol [18] and image analysis software. ${ }^{7}$ The intersection of a line parallel to the spine of the scapula and the line joining the craniocaudal midpoint at the proximal aspect of the humerus and the lateral humeral epicondyle formed the shoulder joint angle. The intersection of the line joining the craniocaudal midpoint at the proximal aspect of the humerus and the lateral humeral epicondyle and the line joining the lateral humeral epicondyle and the craniocaudal midpoint at the distal aspect of the antebrachium formed the elbow joint. The intersection of the line

\footnotetext{
${ }^{1}$ Veterinary Specialty and Emergency Clinic, Philadelphia, PA

${ }^{2}$ MedVet Carmel, Carmel, IN

${ }^{3}$ Sunstone Veterinary Specialists, Portland, OR

${ }^{4}$ DePuy Synthes TPLO, West Chester, PA

${ }^{5}$ Stance analyzer, Companion Animal Health, Newark, DE

${ }^{6} 12$ M-pixel front-facing camera, iPhone 7S, Apple, Cupertino, CA

${ }^{7}$ Adobe Creative Suite, Adobe Systems, San Jose, CA
}

joining the lateral humeral epicondyle and the craniocaudal midpoint at the distal aspect of the antebrachium and a line parallel to the dorsal aspect of the third metacarpal bone formed the carpus. The intersection of the line joining the tuberischiadicum and sacrale and the line joining the greater trochanter and the craniocaudal midpoint at the distal aspect of the femur formed the hip joint angle. The intersection of the line joining the greater trochanter and the craniocaudal midpoint at the distal aspect of the femur and the line joining the craniocaudal midpoint of the proximal portion of the tibia and the lateral malleolus formed the stifle joint. The intersection of the line joining craniocaudal midpoint of the proximal portion of the tibia and the lateral malleolus and a line parallel to the dorsal aspect of the third metatarsal bone formed the tarsus. Hind limb adduction was the angle formed by a line joining the greater trochanter and the center of the metatarsal pad and a vertical line. Left-sided pelvic tilt was the angle formed by a line joining the left and right tuber ischiadicum and a horizontal line. Positive left-sided pelvic tilt meant that the left tuber ischiadicum was lower than the right tuber ischiadicum.

\section{Statistical analysis}

Sample size calculation was based on historic data in dogs with CCL injury [19] and was planned with the intent to detect a differences in mean weight bearing (peak vertical force / body weight) between Amp T- $_{\text {- }}$ PLO and $4 \mathrm{~L}_{\text {TPLO }}$ on the limb that underwent a TPLO at an $\alpha$ of 0.5 and a power $(\beta)$ of 0.8 . Sample size calculation $^{8}$ yield a sample size of 3 to statistically detect a difference of $20 \%$ between groups and a sample size of 9 to statistically detect a difference of $10 \%$ between groups. Statistical analyses were conducted using statistical analysis software. ${ }^{9}$ Due to the small sample size and violation of assumptions of normality and equality of variance, nonparametric tests were used for comparison of variables of interest between groups, including Wilcoxon rank sum tests (Mann-Whitney tests) for comparisons of median values and Spearman's rank correlation coefficients for correlation analyses. Wilcoxon rank sum tests were used to compare joint angles, pelvic tilt, and limb varus for Amp ${ }_{\text {TPLO }}$ and Amp. Since only two amputee dogs were missing a thoracic limb, joint angles were compared only in animals missing a pelvic limb. Correlation analysis was conducted separately for Amp $\mathrm{APLO}_{\mathrm{T} \text { a }}$ and Amp, in regards to

\footnotetext{
${ }^{8}$ https://www.stat.ubc.ca/ rollin/stats/ssize/n2.html, accessed, January 25, 2020

${ }^{9}$ STATA/ IC 14.2, StataCorp LP, College Station, TX
} 

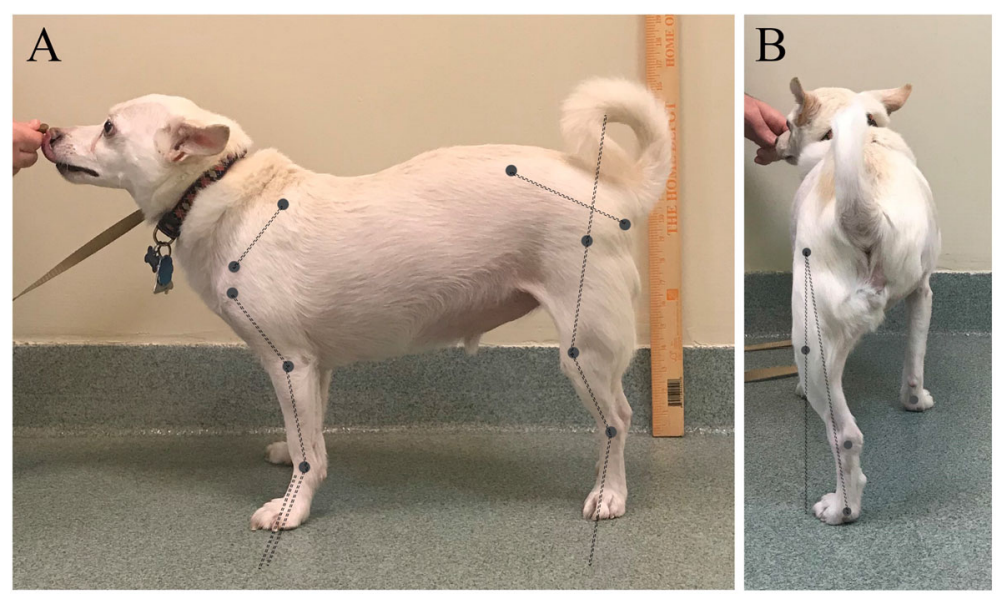

Fig. 2 Pictures acquired from the side (a) and back (b) of an amputee dog who underwent a TPLO 8 weeks earlier. Joints angle are measured by tracing specific anatomic landmarks and drawing lines along or joining those landmarks [18]. For the dog in the picture the shoulder was held at $104.1^{\circ}$, the elbow at $153.1^{\circ}$, the carpus at $213.6^{\circ}$, the hip at $105.1^{\circ}$, the stifle at $146.4^{\circ}$, and the tarsus at $145.1^{\circ}$. Pelvic limb abduction, measured as the angle formed by a vertical line and a line joining the center of the hip joint and metatarsal pad, was $9.1^{\circ}$

percentage of weight bearing in the thoracic limbs, and each of the joint angles for shoulder, elbow and carpus. In dogs missing a hind limb, we tested

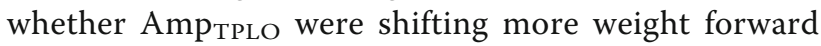
on the side opposite their amputation compared to Amp. For these analyses, side to side data were flipped so that the missing limb was the right hind

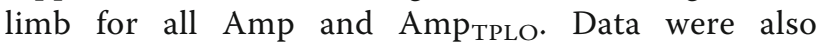
flipped so that all $4 \mathrm{~L}_{\text {TPLO }}$ had TPLO of the left hind

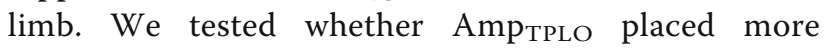
weight on their operated limb compared to $4 \mathrm{~L}_{\text {TPLO }}$

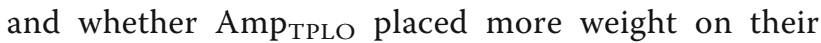
hind limb compared to Amp. For all analyses, statistical significance was $P<0.05$.

\section{Abbreviations}

Amp: Amputees; AmpTPLo: Amputees undergoing TPLO; CCL: Cranial cruciate ligament; 4LTPLO: Four-legged dogs undergoing TPLO; TPLO: Tibial plateau leveling osteotomy

\section{Acknowledgments}

The authors would like to acknowledge the veterinary clinics for their contribution with cases.

\section{Authors' contributions}

RBA conceived the study With DD, DL, and DJML the design, methods, interpretation of data, and drafts of the work were developed. DJML conducted data analysis and prepared illustration. They approved the submitted version and agree both to be personally accountable for the author's own contributions and to ensure that questions related to the accuracy or integrity of any part of the work, even ones in which the author was not personally involved, are appropriately investigated, resolved, and the resolution documented in the literature. AGA contributed with design of the work, data analysis and interpretation and revision of the manuscript. NF helped with data collection. All authors have read and approved the manuscript.

\section{Funding}

Equipment for the study was provided by Companion Animal Health, Newark, DE. The funder did not have any role in study design, collection analysis and data interpretation as well as manuscript writing.

\section{Availability of data and materials}

Additional data would be to offer the spreadsheet that was used for the analyses. It would be the spreadsheet that the statistician used. Raw data with no identifiable information could be available upon reasonable request to the corresponding author.

\section{Ethics approval and consent to participate}

An IRB protocol was not needed because human subjects were not included in our study. This study involved client-owned dogs. An IRB/IACUC protocol was not needed for this study because the data collected were part of the dogs' standard of care. Owners signed an informed consent form to consent to that care. The data were analyzed retrospectively. Any identifiable data from subjects (dogs) were removed before data analysis. A written agreement from each collection site for data sharing was obtained. Data from each collection sites were anonymizes before analysis.

\section{Consent for publication}

The owner of the dog pictured has provided written consent for publication.

\section{Competing interests}

The authors declare that they have no competing interests.

\section{Author details}

${ }^{1}$ Small Animal Orthopedics, Koret School of Veterinary Medicine, Rehovot, Israel. ${ }^{2}$ The Hebrew University of Jerusalem, P.O Box 12, 76100 Rehovot, Israel. ${ }^{3}$ American College of Veterinary Surgeons - Small Animal, Veterinary Orthopedic and Sports Medicine Group, 10975 Guilford Rd Annapolis Junction, Philadelphia, MD 20701, USA. ${ }^{4}$ Department of Physical Therapy, The University of Tennessee at Chattanooga, 615 McCallie Ave Dept \#3253, Chattanooga, USA. ${ }^{5}$ A194 Department of Veterinary Preventive Medicine, College of Veterinary Medicine, The Ohio State University, Columbus, USA. ${ }^{6}$ Veterinary Surgery and Emergency Center, 1114, Philadelphia, South Front Street 19147, USA. ${ }^{7}$ American College of Veterinary Surgeons, Germantown, MD, USA. ${ }^{8}$ American College of Veterinary Sports Medicine and Rehabilitation (Charter), Camas, WA, USA. ${ }^{9}$ Small Animal Orthopedic Surgery, University of California, Davis, USA. 
Received: 28 March 2019 Accepted: 2 June 2020

Published online: 10 June 2020

\section{References}

1. Kirpensteijn J, Van Den Bos R, Endenburg N. Adaptation of dogs to the amputation of a limb and their owners' satisfaction with the procedure. Vet Record. 1999;144:115-8.

2. Withrow SJ, Hirsch VM. Owner response to amputation of a pet's leg. Vet Med Sm Anim Clin. 1979:74:332-4.

3. Raske M, McClaran JK, Mariano A. Short-term wound complications and predictive variables for complication after limb amputation in dogs and cats. J Sm Anim Pract. 2015;56:247-52.

4. Fuchs A, Goldner B, Nolte I, Schilling N. Ground reaction force adaptations to tripedal locomotion in dogs. Vet J. 2014;201:307-15.

5. Kirpensteijn J, Van Den Boss R, Van Den Broom WE, Hazewinkel W. Ground reaction force analysis of large breed dogs when walking after the amputation of a limb. Vet Record. 2000;146:155-9.

6. Hogy SM, Worley DR, Jarvis JC, Hill AE, Reiser RF, Haussler KK. Kinematic and kinetic analysis of dogs during trotting after amputation of a pelvic limb. Am J Vet Res. 2013;74:1164-71.

7. Slocum B, Slocum TD. Tibial plateau leveling osteotomy for repair of cranial cruciate ligament rupture in the canine. Vet Clin North Am Small Anim Pract. 1993;23:777-95.

8. Contreras ET, Worley DR, Palmer RH, Duerr FM. Postamputation orthopedic surgery in canine amputees: owner satisfaction and outcome. Topics Comp Anim Med. 2018:3:89-96.

9. Budsberg SC, Verstraete MC, Soultas-Little R. Force plate analysis of walking gait in the healthy dogs. Am J Vet Res. 1987;48:915-8.

10. Weinstein JI, Payne S, Poulson JM. Use of force plate analysis to evaluate the efficacy of external beam radiation to alleviate osteosarcoma pain. Vet Radiol Ultrasound. 2009;50:673-8.

11. Goldner B, Fuchs A, Nolte I, Schilling N. Kinematic adaptations to tripedal locomotion in dogs. Vet J. 2015;204:192-200.

12. Jarvis SL, Worley DR, Hogy SM, Hill AE, Haussler KK, Reiser RF. Kinematic and kinetic analysis of dogs during trotting after amputation of a thoracic limb. Am J Vet Res. 2013;74:1155-63.

13. Bosscher G, Tomas A, Roe SC, Marcellin-Little DJ, Lascelles DX. Repeatability and accuracy testing of a weight distribution platform and comparison to a pressure sensitive walkway to assess static weight distribution. Vet Comp Orthop Traumatol. 2017;30:160-4.

14. Cole GL, Darryl MD. The effect of limb amputation on standing weight distribution in the remaining three limbs in dogs. Vet Comp Orthop Traumatol. 2017;30:59-61.

15. Clough WT, Canapp SO Jr, Taboada LD, Dycus DL, Leasure CS. Sensitivity and specificity of a weight distribution platform for the detection of objective lameness and orthopaedic disease. Vet Comp Orthop Traumatol. 2018;31:391-5

16. Krotscheck U, Nelson SA, Todhunter RJ, Stone M, Zhang Z. Long term functional outcome of tibial tuberosity advancement vs. tibial plateau leveling osteotomy and extracapsular repair in a heterogeneous population of dogs. Vet Surg. 2016;45:261-8.

17. Feeney L, Lin CF, Marcellin-Little DJ, Tate AR, Queen RM, Yu B. Validation of two-dimensional kinematic analysis of walk and sit-to-stand motions in dogs. Am J Vet Res. 2007;68:277-82.

18. Jaegger G, Marcellin-Little DJ, Levine D. Reliability of goniometry in Labrador retrievers. Am J Vet Res. 2002;63:979-86.

19. Wustefeld-Janssens BG, Pettitt RA, Cowderoy EC, et al. Peak vertical force and vertical impulse in dogs with cranial cruciate ligament rupture and meniscal injury. Vet Surg. 2016;45:60-5.

\section{Publisher's Note}

Springer Nature remains neutral with regard to jurisdictional claims in published maps and institutional affiliations.

Ready to submit your research? Choose BMC and benefit from:

- fast, convenient online submission

- thorough peer review by experienced researchers in your field

- rapid publication on acceptance

- support for research data, including large and complex data types

- gold Open Access which fosters wider collaboration and increased citations

- maximum visibility for your research: over $100 \mathrm{M}$ website views per year

At $\mathrm{BMC}$, research is always in progress.

Learn more biomedcentral.com/submissions 